\title{
Ethnicité, identité ethnique. Quelques remarques pour l'Antiquité tardive
}

\author{
Audrey BECKER \\ Université de Lorraine - Metz \\ audrey.becker@univ-lorraine.fr
}

Recibido: 12 de junio de 2014

Aceptado: 29 de julio de 2014

\section{RÉSUMÉ}

Le concept d'ethnogenèse créé par R. Wenskus puis développé par ses héritiers de l'École de Vienne est depuis quelques années remis en cause par des historiens qui ont, pour certains, déplacé le débat sur le terrain de l'identité ethnique. Mais toutes ces approches présupposent la validité du concept anthropologique d'ethnicité comme élément constitutif et fondamental des identités au $V^{e}$ siècle. Cet article se propose dans un premier temps de revenir, dans une perspective historiographique, sur les différentes définitions de l'ethnicité retenues par les historiens de l'antiquité avant, dans un second temps, de s'interroger sur les sources elles-mêmes pour évaluer si l'ethnicité est réellement un critère premier dans la définition identitaire des nouvelles élites des proto-royaumes barbares de l'Europe du V $V^{\mathrm{e}}$ siècle, particulièrement face aux phénomènes d'acculturation.

Mots clés: Ethnogenèse. Identité ethnique. Ethnicité. Antiquité tardive. Royaumes barbares. Élites aristocratiques.

\section{Ethnicity, ethnic identity. Some remarks for the Late Antiquity}

\begin{abstract}
The concept of ethnogenesis, created by R. Wenskus and developed by his heirs from the Vienna School, has been challenged for the past few years by some scholars who prefer to use the expression «ethnic identity». These theories focus on the concept of ethnicity which is understood as a fundamental element of identities during the fifth century AD. The aim of this paper is to reassess the different definitions of ethnicity which have been used by Late Antique scholars and to question sources in order to evaluate whether ethnicity is really a key criterion for defining aristocratical identity in the European barbarian kingdoms, in particular in relation with the acculturation process.
\end{abstract}

Key words: Ethnogenesis. Ethnic identity. Ethnicity. Late Antiquity. Barbarian kingdoms. Aristocratic elites.

Sommaire: 1. Introduction. 2. Définir l'ethnicité. 2.1. Nécessité épistémologique de définir l'ethnicité. 2.2. Points de repère historiographiques: culturalisme, situationnisme, ethnosymbolisme. 3. L'ethnogenèse et la contextualisation des sources. 3.1. La méthode d'interprétation des sources de H. Wolfram. 3.2 Qu'attendre des sources? 3.3. Redéfinir l'identité barbare, une préoccupation romaine? 4. Conclusion. 


\section{Introduction ${ }^{1}$}

Dans la lignée des approches instrumentalistes de l'ethnicité développées à partir des années 1950-1960, R. Wenskus puis ses héritiers de l'École de Vienne, H. Wolfram ou encore W. Pohl sont les premiers à avoir tiré profit pour l'histoire de l'Antiquité Tardive de la notion anthropologique d'ethnicité. ${ }^{2} \mathrm{R}$. Wenskus a, en effet, établi un modèle selon lequel les barbares entrés dans l'Empire romain au $V^{e}$ siècle n'étaient pas des peuples mais des armées polyethniques dirigées par des rois. Elles auraient acquis une identité ethnique avant tout grâce à une manipulation de l'idéologie et du discours politique par les rois et leur aristocratie, manipulation basée sur la création de mythes auxquels auraient adhéré des groupes ethniquement hétérogènes. ${ }^{3} \mathrm{H}$. Wolfram postule ainsi qu'on retrouve des traces de ces mythes dans les récits dits «des origines» des différents peuples. Ils seraient, au moins pour partie, construits autour de faits historiques qu'une analyse ethnographique permettrait de mettre à jour. ${ }^{4}$ Cette théorie a été à l'origine de nombreux débats et de remises en cause parfois virulentes notamment chez les historiens anglo-saxons. ${ }^{5}$ Récemment ces questions ont donné lieu en France, en Espagne ou en Italie à une relecture plus nuancée des thèses de l'École de Vienne. ${ }^{6}$ Il ne s'agit pas dans ces quelques pages de prétendre résoudre tous les problèmes liés à l'ethnogenèse mais simplement d'en évoquer certains qui paraissent essentiels car mettant en jeu les éventuelles limites conceptuelles de cette théorie. En effet, si personne ne songe plus sérieusement à remettre en cause l'idée que les groupes barbares ne sont pas déterminés biologiquement, ${ }^{7}$ l'utilisation des sources faite par les tenants de l'ethnogenèse soulève certains problèmes fondamentaux renvoyant aussi bien à des questionnements historiques qu'anthropologiques. Surtout, cette approche présuppose la validité du concept d'ethnicité comme élément constitutif et fondamental aux yeux mêmes des élites barbares. Encore faut-il le définir et en démontrer la validité opératoire au $\mathrm{V}^{\mathrm{e}}$ siècle.

1 Ce texte est la version écrite d'une conférence donnée dans le cadre du séminaire de recherche doctorale de la faculté des lettres de l'Université du Pays Basque à Vittoria le 25 avril 2012 organisé par Elena Torregaray. Qu'elle soit ici remerciée pour son invitation et son hospitalité. Les abréviations des sources suivent celles de Hornblower - Spawforth (eds.), 2012, ou à défaut celles de Martindale 1980-1992. Les abréviations des revues reprennent celles de l'Année Philologique (http://www.annee-philologique.com/files/sigles_fr.pdf).

2 Sur la notion d'ethnicité, A. D. Sмiтн 1986; ID. 1994.

3 Wenskus 1961; Wolfram 1970; Pohl 1992; Pohl (ed.), 1997; Pohl - Reimitz (eds.), 1998.

4 WOLFRAM 1990; ID. 1990a.

5 Gillett (ed.), 2002; Geary 2003.

6 Gazeau - Bauduin - Modéran (eds.), 2008; Brogiolo - Chavarría Arnau 2005; on peut, par ailleurs, noter le projet mené conjointement par l'université de Madrid et la casa de Velásquez ayant abouti à la tenue d'un colloque en octobre 2013, Identity and Otherness among the Barbarians in Late Antiquity and Early Medieval Europe, The difficult relationship between textual and archaeological record dont les actes ne sont pas encore à ce jour publiés.

7 Dans la thèse primordialiste, l'individu naît avec les éléments constitutifs de son identité ethnique (langage, religion, phénotype...). Son appartenance au groupe ethnique lui est donc imposée par la force coercitive des liens du sang. À l'origine de cette théorie, Shils 1957; Kallen 1924; Geertz 1963. Cf. Van den Berghe 1970 à l'origine du paradigme sociobiologique de l'ethnicité qui, dans la continuité des approches racistes du primordialisme, affirme que les bases de l'ethnicité sont à trouver dans les prédispositions génétiques d'un groupe à se reproduire pour assurer sa survie. 


\section{Définir l'ethnicité}

\subsection{Nécessité épistémologique de définir l'ethnicité}

Définir l'ethnicité est une nécessité pour tout historien qui veut utiliser valablement ce concept anthropologique en le confrontant aux sources historiques et déterminer si ce qu'elles nous donnent à connaître de l'histoire des peuples barbares relève ou non du domaine de l'ethnicité. Or, paradoxalement, si le concept d'ethnicité est régulièrement évoqué pour expliquer des phénomènes anthropologiques de l'Antiquité Tardive ou du Haut Moyen Âge chez les partisans des théories de l'ethnogenèse comme chez ses détracteurs, on n'en trouve que rarement une définition précise. ${ }^{8}$ Tout se passe comme si le sens de ce concept allait de soi et n'avait donc pas besoin d'être précisé ou à l'inverse, qu'il ne devait l'être qu'au terme d'une enquête qui précisément postule a priori sa validité, ce qui épistémologiquement est pour le moins discutable.

La définition anthropologique du groupe ethnique fait aujourd'hui l'objet d'un large consensus et se réfère explicitement aux définitions des anthropologues américains des années 1970 qui, les premiers, ont cessé d'envisager le groupe ethnique comme un groupe identifiable objectivement par sa culture. Il est aujourd'hui défini comme

un groupe humain déterminé, partageant une généalogie commune mythique, une mémoire historique, des éléments de culture, un lien avec une patrie propre, et un sens de solidarité. ${ }^{9}$

Quant au concept d'ethnicité lui-même, s'il reste toujours chez les anthropologues des points de débats, néanmoins un consensus se dégage autour d'une définition a minima qui l'envisage comme un processus relationnel de dichotomisation entre membres et non-membres qui s'identifient et sont identifiés à travers l'interaction sociale. ${ }^{10}$ Le processus d'ethnicité est donc un phénomène social et la mobilisation de certains traits culturels propres à un groupe ethnique et supposés venir de l'origine commune réelle ou fictive permet le maintien de la frontière entre «Nous» et «Eux», entre membres et non-membres. Ces traits culturels sont alors soit revendiqués soit niés quand les groupes ethniques définissent leur Identité tout en définissant l'Altérité dans une démarche à la fois dialectique mais aussi subjective quant au choix

\footnotetext{
8 On cherchera par exemple en vain une définition de l'ethnicité dans les différents travaux de H. Wolfram. Les anthropologues eux-mêmes ont longtemps contourné le problème en posant comme objet d'étude l'ethnicité sans pour autant la définir. IsAJI 1974 soulignait ainsi ce problème dès les années 1970 après avoir relevé que dans 65 études portant sur l'ethnicité, 52 n'en proposaient aucune définition.

9 A. D. Smith 1993, 130; voir également Glazer - Moynihan (eds.), 1975; De Vos - Romanucci-Ross (eds.), 1975.

10 Sur cette question, voir en particulier l'introduction de BARTH 1969; également JUTEAU 1999 qui reprend et approfondit les travaux de F. Barth; BASTENIER 2004.
} 
des éléments qui vont nourrir le sentiment d'identité et d'appartenance à un groupe ethnique. $^{11}$

\subsection{Points de repère historiographiques: culturalisme, situationnisme, ethnosymbo- lisme}

P. Bauduin, dans un ouvrage récent sur la question, reprend cette définition comme base de sa réflexion en affirmant en particulier le caractère relationnel, dynamique et construit de l'ethnicité. ${ }^{12}$ Mais les débats qui agitent encore les anthropologues autour de certains aspects de la définition de l'ethnicité se retrouvent dans les positions parfois adoptées par des historiens autour de ce problème.

Certains s'appuient ainsi encore sur des postulats clairement culturalistes. C'est par exemple le cas de R. Pallas Brown qui ne laisse pas de surprendre lorsqu'elle définit l'ethnicité comme étant statique et résistante au changement, tout en précisant dans le même temps que les groupes ethniques réagissent aux changements sociaux politiques ou économiques pour créer ou recréer leur identité. ${ }^{13}$ Surtout, l'ethnicité se définit, selon elle, essentiellement comme une série de caractéristiques religieuses, sociales, politiques, morphologiques, linguistiques et/ou culturelles. Elle rejoint ainsi la définition posée par G. Greatex qui voit l'ethnicité comme la somme de critères raciaux, biologiques, linguistiques, religieux, territoriaux, même s'il admet, par ailleurs, que l'ethnicité est subjective et résulte du regard d'un groupe sur lui-même et sur les autres groupes. ${ }^{14}$ Cette vision se situe clairement dans la lignée de l'approche culturaliste de l'ethnicité telle qu'elle a été définie dans les années 1950 par les Cross-Cultural Surveys particulièrement par R. Naroll. ${ }^{15}$ Elle pose le problème de la définition d'une identité ethnique à partir d'éléments matériels à l'image de ce qu'ont par exemple fait les archéologues et les anthropologues de l'ex-URSS en associant les Slaves à la culture de Chernyakhov, ou encore les archéologues allemands autour des travaux portant sur les vêtements identifiés comme marqueurs ethniques. ${ }^{16}$

Or, certains éléments de cette approche ont été largement remis en cause. Les anthropologues E. Leach mais aussi F. Barth ou encore M. G. Smith ont montré, pour certains dès les années 1950, qu'il était impossible d'établir d'une part une liste

11 Poutignat - Streiff-Fenart 1995, 134.

12 Bauduin 2008.

13 Pallas-Brown 2000. Par ailleurs, la référence faite à F. Barth, à propos de la possibilité grâce au primordialisme d'envisager l'ethnicité comme universelle, est assez curieuse. En tout état de cause, sa vision de l'ethnicité parait confuse.

14 Greatrex 2000.

15 Naroll 1964 crée le concept d'unités culturelles, les cult-units, qu'il définit comme l'ensemble des personnes parlant la même langue, habitant sur un même territoire et appartenant à la même communauté politique. Surtout, il envisage les cults units comme des isolats limités par l'impossibilité d'établir une intercommunication avec les autres cult units.

16 Pour une présentation des travaux des archéologues de l'ex-URSS, CURTA 2002, particulièrement 210212. Pour l'utilisation de la culture matérielle comme moyen d'identifier des populations ethniquement, ZEISS 1931, ou plus récemment, SIEGMund 1996 qui a utilisé aussi bien les habits que la vaisselle et l'armement retrouvés dans des tombes pour identifier différents modèles de culture correspondant, selon lui, à différents groupes ethniques barbares. Sur ce point, également SiEGMund 1999. 
exhaustive de critères «culturels» pour délimiter les groupes ethniques et, d'autre part, un lien entre culture matérielle et ethnicité. ${ }^{17}$ De plus, d'un point de vue historiographique les théories des ethnographes de l'ex-URSS sont largement liées à l'idéologie de l'ethno-nationalisme. ${ }^{18}$ Surtout, ces théories partent du présupposé que les éléments matériels d'une culture sont nécessairement des marqueurs ethniques en n'envisageant absolument pas que ceux-ci puissent être des marqueurs sociaux ou politiques. D'autre part, en ayant une vision statique de l'ethnicité, elles ne s'intéressent pas, par définition, aux mouvements d'acculturation possibles. C'est d'ailleurs l'un des reproches faits par S. Brather à ces théories: la présence de fibules franques chez les Alamans peut certes être interprétée comme une preuve de la présence franque mais elle peut tout aussi bien témoigner d'une acculturation d'une partie des Alamans qui pouvaient ainsi manifester concrètement leur allégeance aux Francs. ${ }^{19}$

À l'exact opposé de ces théories et dans la continuité des théories de l'École de Vienne, les travaux de P. Geary ou de P. Heather sont organisés autour d'une approche totalement utilitariste et situationniste où l'ethnicité est un instrument au service des intérêts d'un groupe. ${ }^{20} \mathrm{P}$. Heather pense l'ethnicité comme totalement dépendante du contexte politique et manipulée par les acteurs pour imposer leur pouvoir. L'identité ethnique aurait donc un aspect artificiel et serait une donnée construite et subjective. Dès lors, se pose la question de ce que les anthropologues nomment la saillance: l'ethnicité devient une identification possible parmi d'autres pour les acteurs qu'ils choisiraient ou non de mettre en avant en fonction des circonstances. ${ }^{21}$ On ne peut que constater la parenté intellectuelle avec des anthropologues tels que O. Patterson ou encore M. Moerman. ${ }^{22}$ Cette approche pose, par ailleurs, la question de l'invariante

17 LEACH 1954 pour une critique point par point de la théorie de Naroll; dans son étude sur les Lue en Thaïlande, Moerman 1968 remet totalement en cause le concept d'isolat ethnique; quant à M. G. SMITH 1982, particulièrement 2 et 11-12, il fait une critique très poussée de la Harvard Encyclopedia of the American ethnic Groups publiée en 1980 qui identifie, en appliquant les principes développés par Naroll, 101 groupes ethniques aux Etats-Unis d'Amérique à partir de 14 caractéristiques telles que la langue, les préférences alimentaires, la race, la religion, l'intérêt pour la politique de leur pays d'origine ou encore la conscience interne au groupe de sa différence ethnique. M. G. Smith dénonce des résultats parfois très surprenants dans la délimitation des groupes ethniques, toutes les tribus indiennes américaines étant, par exemple, rassemblées dans un seul groupe ethnique, à l'instar d'ailleurs de tous les américains originaires des Iles du Pacifique ou encore d'Afrique.

18 Dragadze 1980. Sur l'ethno-nationalisme: Shanin 1989; Tishrov 1994.

19 BRATHER 2002 très critique contre Koch 1997. Sur la polémique entre Brather et Curta quant à la possibilité ou non d'interpréter ethniquement la culture matérielle, voir en dernier lieu CURTA 2013. Sur cette question, voir également le travail de Halsall 2007 et plus récemment, Halsall 2011 réfutant point par point l'idée défendue par Kazanski et Perrin que le matériel archéologique funéraire permet une identification ethnique. KAZANSKI - PÉRIN 2008.

20 Geary 1983; Heather 1996; ID. 2003.

21 C'est pour cette raison, selon lui, que les aristocrates ostrogoths installés dans le nord de l'Italie refusent la proposition de Bélisaire, c'est-à-dire la pleine propriété de leur terre contre l'arrêt des combats. Ce refus s'expliquerait par la peur de perdre ce qui fonde leur supériorité politique, à savoir leur identité ethnique. Ce serait cet intérêt politique qui les aurait poussés à continuer la guerre qui ne s'achèverait que faute de combattants, une fois l'élite aristocratique décimée, les autres Ostrogoths sans pouvoir politique à défendre ayant alors rendu les armes.

22 Moerman 1968 pour qui il ne s'agit pas de définir l'ethnie Lue mais plutôt de savoir autour de quels enjeux cette identification ethnique «Lue» est préférée à d'autres possibles. 
importance de l'ethnicité dans les phénomènes identitaires. Les études de O. Patterson sur les communautés jamaïcaines ou portoricaines aux États-Unis montrent ainsi très clairement que l'ethnicité est une identification possible parmi d'autres (religion, sexe, classe sociale, lieu de résidence...) à disposition des Portoricains et qu'ils décident ou non de revendiquer en fonction de leurs intérêts. ${ }^{23}$ Cette approche de l'ethnicité n'est pas sans rappeler l'explication fournie par P. Amory à propos de la disparition des Ostrogoths en Italie après leur défaite contre les armées de l'Empire romain d'Orient: ils auraient abandonné cette identification ethnique parce qu'elle ne pouvait plus rien leur apporter. Mieux encore, il s'applique à démontrer que les Ostrogoths, une fois l'identification ethnique abandonnée, se seraient tout simplement intégrés aux armées byzantines. ${ }^{24}$ P. Amory franchit une étape supplémentaire car il est un des premiers à postuler que, si les Ostrogoths ont pu abandonner l'identification ethnique aussi rapidement, c'est bien parce qu'il ne s'agissait que d'une identification et non pas d'une identité ethnique.

Enfin, il faut s'arrêter sur la position de W. Pohl qui, tout en se plaçant dans la lignée des héritiers de l'École de Vienne, a repensé la notion d'ethnogenèse et, en corollaire, celle d'ethnicité. ${ }^{25} \mathrm{Il}$ a ainsi déplacé sa réflexion sur le terrain de l'identité ethnique en reprenant tout d'abord à son compte les travaux de l'anthropologue A. D. Smith, particulièrement l'idée que le concept d'ethnicité s'applique à toutes les périodes historiques. ${ }^{26} \mathrm{Il}$ faut d'ailleurs souligner qu'il est l'un des rares historiens à sortir de l'implicite autour de ces questions et à poser clairement les références anthropologiques qui servent de cadre à ses analyses. ${ }^{27}$ Ainsi, s'il rejette les théories culturalistes, ${ }^{28}$ en revanche il ne refuse pas a priori l'idée que l'ethnicité puisse être liée «aux affects primordiaux» tels que les décrit $\mathrm{C}$. Geertz dans une approche quelque peu néo-primordialiste..$^{29} \mathrm{Il}$ s'élève également contre les visions trop strictement utilitaristes de l'ethnicité qui finissent par la vider complètement de tout contenu culturel. Surtout, en adoptant à la suite d'A. D. Smith, une perspective ethnosymbolique, ce sont les symboles identitaires autour de la croyance en une origine commune-souvenirs et mythes- qui prennent pour $\mathrm{W}$. Pohl une importance toute particulière car ils sont vus et analysés comme les supports concrets du discours ethnique toujours envisagé comme la base de la construction politique et identitaire des peuples barbares. Ce qui fonde l'ethnie pour W. Pohl, c'est la croyance en une origine commune. Et tous les marqueurs ethniques ne sont pertinents en tant qu'élément de définition du groupe

23 Patterson 1975. Il faut souligner le lien avec les théories sociologiques individualistes méthodologiques développées par R. Boudon contre le structuralisme de P. Bourdieu ou encore avec les théories du choix rationnel developpées de J. Coleman. Voir Boudon 1979; ID. 2002; Coleman 1994.

24 AMORY 1997, 317.

25 PoHL 2002, particulièrement 224-225.

26 Sur la question de la pérennité du concept d'ethnicité, A. D. SмITH 1981, 86; voir aussi Armstrong 1982 et le compte rendu de cet ouvrage de A. D. SмIтн 1984.

27 Pour les références à A. D. Smith, PoHL 2005, particulièrement 185; ID. 2002, 221-239, 228, 235.

28 Sur les théories culturalistes, POHL 2006.

29 Pohl 2005, 195. Pour GeERTz 1963, les affects primordiaux dériveraient d'un sentiment d'affinité naturelle reposant sur des liens intuitivement perçus comme immédiats et naturels (lien du sang, religion, langue...), constitutifs de l'identité ethnique. 
ethnique qu'à partir du moment où ils justifient le mythe de l'origine commune qui devient pour reprendre l'expression de P. Ricoeur un «mythomoteur». ${ }^{30}$

$\mathrm{Au}$ final, ce rapide survol historiographique, nécessairement schématique et non-exhaustif, fait apparaître qu'il y a autant d'utilisations possibles du concept d'ethnicité que de théories anthropologiques autour de ce terme. Ce foisonnement, s'il complique les premiers repérages de l'historien s'aventurant dans ce domaine, ouvre de vraies perspectives de travail sur la question du phénomène ethnique pour l'Antiquité Tardive et le Haut Moyen Âge, à la condition cependant d'avoir conscience des présupposés anthropologiques des différentes approches. Mais les derniers travaux de W. Pohl autour des questions d'identification sont peut-être les plus ambitieux et les plus novateurs épistémologiquement. Aux théories instrumentalistes et situationnistes développées particulièrement chez les historiens anglo-saxons, W. Pohl, en reprenant à son compte les critiques faites aux théories de l'ethnogenèse défendues par R. Wenskus et $\mathrm{H}$. Wolfram après lui, répond en adoptant l'utilisation d'un autre concept que celui d'ethnogenèse, à savoir celui de construction de l'identité ethnique. ${ }^{31}$ Cela lui permet de ne pas laisser de côté certains aspects de l'ethnogenèse et, dans le même de temps, d'envisager l'identité ethnique en relation avec d'autres identités possibles (culturelles, religieuses, sociales, économiques...). Surtout, il abandonne l'idée chère à $\mathrm{H}$. Wolfram selon laquelle on pourrait grâce à l'étude des récits des origines atteindre à la connaissance historique des origines des gentes barbares. $^{32}$

\section{L'ethnogenèse et la contextualisation des sources}

\subsection{La méthode d'interprétation des sources de H. Wolfram}

La méthode d'interprétation des sources développée en particulier par H. Wolfram est pour le moins problématique car il s'autorise à passer outre les règles habituelles de la critique textuelle. À propos de l'origo gentis des Goths, il écrit ainsi:

Et cela ne fait pas beaucoup de différences que cette littérature viennent seulement du milieu du sixième siècle et le plus souvent provienne des huitième, neuvième ou dixième, jusqu'au douzième siècle. ${ }^{33}$

Dès lors, il s'appuie aussi bien sur Jordanès que sur des sources datant au moins du $\mathrm{X}^{\mathrm{e}}$ siècle comme Widukind ou les Niebelungen pour étayer sa théorie de la création de mythes communs par les rois barbares, y puisant tous les éléments d'apparence ethnographique qui confortent son modèle et faisant de chacune d'entre elles

\footnotetext{
30 Sur l'importance de l'origine commune dans les approches anthropologiques: PouTiGNAT 1995, 175-181.

31 Il est ainsi remarquable que dans PoHL 2008, 23, «ethnogenèse» ne figure pas dans les mots clés contrairement aux entrées «identité ethnique» et «construction identitaire».

32 Cf. PoHL 2005, particulièrement 200-201.

33 Wolfram - Pohl (hrsg.), 1990, 617.
} 
un miroir de la transmission orale du noyau de tradition des peuples barbares. Mais c'est faire peu de cas du contexte de rédaction de ces sources et oublier un peu rapidement que ce qu'elles permettent d'atteindre, c'est le regard d'un individu situé historiquement. ${ }^{34}$ Surtout, cette approche téléologique aboutit à une surinterprétation des sources puisqu'elles sont lues selon une théorie modèle à laquelle elles doivent nécessairement fournir des éléments. Or, le travail de contextualisation des sources est une nécessité fondamentale ${ }^{35}$ sous peine d'aboutir au mieux à une mauvaise compréhension des sources, au pire à des contresens, particulièrement pour les sources latines du $V^{e}$ siècle. C'est ainsi le cas à différentes reprises lorsque H. Wolfram fait référence aux lettres de Sidoine Apollinaire. À propos d'une lettre envoyée en 469 à Syagrius, alors conseiller du roi burgonde Gondebaud, ${ }^{36}$ il écrit:

Sidoine Apollinaire remarque en plaisantant que les Burgondes craignaient de faire des barbarismes dans leur propre langue barbare devant des aristocrates romains, qui connaissaient maintenant cette dernière à la perfection. ${ }^{37}$

Or il ne s'agit pas pour Sidoine d'une plaisanterie, bien au contraire, mais d'une critique très acerbe de Syagrius pour sa connaissance de la langue burgonde, ce qui laisse entendre qu'il ne s'agissait pas encore en 469 d'une situation normale et admissible pour un aristocrate. ${ }^{38}$

\subsection{Qu'attendre des sources?}

Les travaux de M. Coumert sur les récits des origines des peuples prennent le contrepied de la méthode de $\mathrm{H}$. Wolfram en analysant ces récits après les avoir resitués dans leur contexte de rédaction. Sa démarche scientifique est claire: ne pas postuler a priori qu'il s'agit de récits ethnographiques mais faire la part de ce qui renvoie aux sources écrites antérieures et de ce qui fait réellement référence à des traditions orales

\footnotetext{
34 Sur cette question, Gillett 2002; Goffart 1988; ID. 2002; McKitterick 2004; Carruthers 1990.

35 Sur l'importance de la contextualisation, GoFFART 1988, 329-431.

36 Sid. Apoll. Epist. V, 5, 3: quod te praesente formidet linguae suae facere barbarus barbarismum. Adstupet tibi epistulas interpretanti curua Germanorum senectus et negotiis mutuis arbitrum te disceptatoremque desumit. Nouus Burgundionum Solon in legibus disserendis... «...en ta présence, le barbare redoute de faire un barbarisme dans sa propre langue. Le conseil des vieillards germains, courbés par les ans est stupéfait de te voir traduire ses lettres et te prend comme arbitre et comme juge dans les affaires communes. Nouveau Solon des Burgondes dans l'interprétation des lois...».

37 WOLFRAm 1990, 29.

38 Il faut d'ailleurs souligner la différence de ton employé par Sidoine lorsqu'il écrit à Léon, un autre aristocrate gallo-romain conseiller du roi wisigoth Euric. Alors qu'il est pour le moins ironique à propos de Syagrius, en particulier lorsqu'il le félicite pour sa parfaite maîtrise de la langue burgonde, le ton qu'il emploie dans les lettres adressées à Léon est au contraire laudatif (en particulier Sid. Apoll. Epist. VIII, 3, 3). Cette différence résulte des circonstances politiques dans lesquelles Sidoine s'est adressé à chacun d'eux. Lorsqu'il écrit à Syagrius, au plus tard en 469, Sidoine ne se sent probablement pas encore menacé politiquement par les Burgondes, alors qu'en 476, lorsqu'il écrit à Léon la première des deux lettres qu'il lui a adressées, l'empereur d'Occident a alors reconnu l'indépendance des royaumes wisigoth et burgonde (Ennod. Vita Epiph. 85-89; Sid. Apoll. Epist. VII, 6, 10; Sid. Apoll. Epist. VII, 7, 4) et Sidoine doit bien alors composer avec les nouveaux maîtres de la Gaule.
} 
propres aux différents groupes barbares. ${ }^{39}$ C'est d'un point de vue méthodologique une avancée majeure, en particulier autour de l'étude d'une œuvre qui a fait et fait encore débat parmi les historiens: les Getica de Jordanès. Sans revenir ici trop avant sur les détails, il faut néanmoins rappeler l'enjeu historiographique posé par cette source. Les historiens allemands et autrichiens, reprenant les perspectives développées par A. Momigliano, ont systématiquement minoré le rôle joué par Jordanès qui n'aurait été d'après eux qu'un abréviateur et non le réel auteur des Getica,${ }^{40}$ point de vue largement rejeté par les historiens anglo-saxons pour qui Jordanès est véritablement l'auteur de cette source ${ }^{41}$ En réalité, l'enjeu historiographique dépasse largement la simple querelle autour de la paternité de cette œuvre car R. Wenskus, et après lui H. Wolfram, ont fait de cette source le cœur de leur théorie de l'ethnogenèse. En effet, en postulant que derrière les Getica se trouve en réalité l'œuvre de Cassiodore, ils font de cette source le premier récit des origines à destination d'un peuple barbare et non plus des lettrés de l'Empire romain. Dès lors, $\mathrm{H}$. Wolfram affirme la nécessité de déstructurer l'œuvre de Jordanès. Il faudrait selon lui:

mettre la structure historique de Cassiodore sens dessus dessous, afin que l'origo gothica, l'origine des Goths eux-mêmes, réémerge de l'historia romana ${ }^{42}$

Mais l'analyse précise menée par M. Coumert fait apparaitre qu'il ne s'y trouve au final que très peu d'éléments renvoyant aux traditions gothiques. Surtout, il paraît difficile aujourd'hui de continuer à nier le rôle de Jordanès dans l'écriture ou la réécriture de l'œuvre. Dans tous les cas, il est pour ainsi dire impossible de déterminer précisément ce qui vient de l'un ou de l'autre. ${ }^{43}$ Et quand bien même on accepterait avec H. Wolfram l'idée que Théodoric a voulu, à travers les écrits de Cassiodore simplement compilés par Jordanès, créer des mythes fondateurs, cette origo gentis resterait le fait d'un homme s'exprimant en latin et manipulant des concepts ethniques liés à sa culture et qu'on ne peut donc saisir qu'à travers le filtre culturel romain. ${ }^{44}$ Connaître les limites inhérentes à la nature même des sources de l'Antiquité Tardive et les accepter est fondamental: les sources sont jusqu'au milieu du $\mathrm{VI}^{\mathrm{e}}$ siècle un miroir plus ou moins déformant de la réalité barbare qui ne nous est accessible qu'à travers une interpretatio romana ${ }^{45}$ Avoir conscience de cela c'est accepter du même coup de remettre en cause la validité des grilles de lecture qui leur ont généralement été appliquées jusqu'à présent: il n'existe pas de discours ethniques barbares en-

39 COUMERT 2007, 28; ID. 2008.

40 Momigliano 1955.

41 Bradley 1966; Reydellet 1981; Goffart 2006, 56-72 qui n’hésite pas à faire de Jordanès un agent au service des Byzantins et de leur politique de destruction du royaume ostrogothique, ce qui parait un peu excessif.

42 Wolfram 1990, 15-16.

43 Coumert 2007, 45-52, 125-142.

44 C'est peut-être pour contourner ce problème qu' $\mathrm{H}$. Wolfram affirme, sans aucune preuve, que Théodoric aurait dicté à Cassiodore son œuvre, à l'origine des Getica de Jordanès, la forme la plus aboutie, selon lui, de manipulation des mythes par un roi. Cf. WOLFRAM 1990, 324.

45 Sur cette question, Hummer 1998, 27, n. 8, qui affirme de façon pour le moins surprenante que «les observations d'Ammien amènent le lecteur derrière l'interpretatio romana dans le monde barbare». 
dogènes pour l'Antiquité Tardive avant la seconde moitié du VI ${ }^{\mathrm{e}}$ siècle. Ce que les sources littéraires romaines nous donnent avant tout à connaître, c'est la perception que les Romains avaient des Barbares, de leur organisation politique, de leur ethnicité, exprimée à l'aide d'une terminologie latine, ou grecque, liée à la représentation qu'ils se faisaient de ces Barbares.

\subsection{Redéfinir l'identité barbare, une préoccupation romaine?}

La reconnaissance à partir du $\mathrm{V}^{\mathrm{e}}$ siècle, aussi bien politique que diplomatique, des gentes par les Romains a provoqué un embarras manifeste dans les sources autour de l'utilisation du terme même de barbarus car l'identité romaine s'était construite jusque là autour du rejet du barbarus, défini comme l'image négative de l'Altérité, s'opposant à la romanitas et aux valeurs qu'elle véhicule. Or, reconnaître une existence politique et diplomatique à ces gentes, c'est précisément faire entrer dans la romanité son exacte antithèse. Ces gentes ne peuvent être considérées comme barbares, avec tout ce qu'implique ce concept, et en même temps être reçues comme des interlocutrices politiques valables.

Face à cela, s'est donc imposée la nécessité de leur donner une identité politique acceptable dans le cadre de la romanité en faisant notamment évoluer le sens et l'utilisation du vocabulaire politique traditionnellement attaché aux peuples barbares, qu'il s'agisse de gens ou de rex, pour leur faire perdre progressivement leur caractère barbare. C'est ainsi que si au IV siècle gens renvoie encore aux barbares de façon indifférenciée sans existence juridique à l'extérieur de l'Empire romain, au cours du $V^{\mathrm{e}}$ siècle, l'emploi et la signification même de ce terme évoluent vers le sens de communauté politique. ${ }^{46} \mathrm{D}$ 'autre part, seuls les rois barbares auxquels les Romains sont confrontés dans les différentes provinces de l'Empire d'Occident voient leur titulature évoluer d'un modèle où le nom de la gens qualifie le roi, vers l'utilisation sans autre qualificatif de rex, employé dès lors comme un véritable élément de titulature que les Romains n'hésitent pas à mettre en parallèle avec imperator ${ }^{47}$ La distinction ethnique pour qualifier un rex n'existe finalement que comme un premier moyen

46 À la suite de l'usage introduit par Tacite, si Ammien Marcellin emploie encore gentes sous la forme d'un pluriel indifférencié (Amm. Marc. XIV, 3, 1; XVII, 3, 1; XIX, 1, 8; XXII, 8, 43; XXII, 5, 66; XXIII, 6, 83), l'usage du singulier ou du pluriel précisé par le nom du peuple au génétif apparaît (XV, 12, 5; XXIII, 6, 13; XXVII, 4, 11; XXIII, 6, 13; XXVII, 4, 11; XXVI, 6, 11; XXXI, 2, 1; XXXI, 9, 5) permettant ainsi une première individualisation de ces groupes. Au début du VI ${ }^{\mathrm{e}}$ siècle, cette individualisation des communautés politiques barbares aboutit dans les écrits de Cassiodore à la mise en place d'un véritable vocabulaire juridique et abstrait pour qualifier les gentes (Cassiod. Var. I, 1, 1: utilitas gentium; III, 23, 3: virtutes gentium; III, 3, 2: leges gentium; X, 31, 4: gentis utilitatem; XI, 13, 4: gentibus virtute; VI, 3, 6: legatos gentium; VII, 33, 1: gentis legati; XII, 4, 1: legatis gentium).

47 Hyd. Lem. 170: Per Augustum Avitum Fronto comes legatus mittitur ad Sueuos. Similiter et a rege Gothorum Theodorico, quia fidus Romano esset imperio, legati ad eosdem mittuntur. «Le comte Fronto est envoyé par l'Auguste Avitus en ambassade auprès des Suèves. En même temps, Théodoric, le roi des Goths, fidèle allié de l'Empire romain, leur envoie une ambassade»; 197: Legati, a Nepotiano magistro militiae et a Sunerico comite missi ueniunt ad Gallaecos nuntiantes Maiorianum Augustum et Theodoricum regem firmissa inter se pacis iura sanxisse, Gothis in quodam certamine superatis. «Des ambassadeurs envoyés par Nepos, maître de la milice, et par le comte Sunéric viennent annoncer aux Galiciens que Majorien Auguste et le roi Théodoric ont conclu et juré entre eux une paix très solide, après une certaine défaite des Goths». 
commode d'identification des rois barbares qui disparaît quand le rex devient un véritable interlocuteur politique bien individualisé. Cette évolution reflète donc avant tout des usages romains et non un discours barbare sur l'identification ethnique. ${ }^{48} \mathrm{Du}$ même coup, l'évolution de la titulature traduit une perte d'identité barbare avec ce que cela inclut de péjoratif pour un Romain.

La question qui reste posée est celle de la réalité de la préoccupation ethnique au $V^{e}$ siècle pour les rois barbares eux-mêmes dans la construction de leurs royaumes. Sur cette question, P. Amory répond très clairement dans ses différents travaux: la construction de l'identité barbare ne se fait pas, selon lui, autour de critères ethniques. Concernant les Ostrogoths, elle se ferait autour de critères géographiques et socio-économiques ${ }^{49}$ et ce que les historiens ont pris pour une solidarité ethnique autour de Théodoric relèverait, en réalité, de l'une allégeance politique. Et de fait, clairement, cette allégeance politique n'implique pas au V $\mathrm{V}^{\mathrm{e}}$ siècle, d'abandonner son identité ethnique pour adopter celle du détenteur de l'autorité. L'identité ethnique ne préjuge pas de la loyauté politique: l'entourage des rois barbares, mais aussi plus largement la population de leur royaume, est clairement polyethnique. En réalité, les problèmes ne surgissent qu'à partir du moment où deux identités dans lesquelles peut se reconnaitre un même homme entrent en conflit. ${ }^{50}$

Mais ce qui est encore plus intéressant pour le $\mathrm{V}^{\mathrm{e}}$ siècle, c'est la part prise par les aristocrates gallo-romains ou italiens dans les constructions identitaires des élites barbares. Confrontés au problème fondamental, pour leur propre survie en tant qu'élite, de la nécessité d'une redéfinition tenant compte de ces nouveaux acteurs politiques et diplomatiques que sont les élites barbares, les aristocrates romains réussissent à maintenir la culture latine comme élément discriminant positif, à la fois pour euxmêmes mais aussi pour les élites barbares toujours en quête d'une légitimité toute tournée vers cette romanité culturelle à laquelle elles accèdent précisément grâce aux Romains qui gravitent dans leur entourage ${ }^{51}$ C'est ainsi que Théodoric II choisit Avitus comme précepteur de ses enfants ou que Gondebaud participe à des discussions théologiques. ${ }^{52}$ En réalité l'enjeu culturel pour les rois barbares est clairement défini par Cassiodore: la suprématie politique.

Spernenda non sunt quae a uicinis regibus praesumptionis gratia postulantur, dum plerumque res paruae plus praeualent praestare quam magnae possunt optinere diuitiae. Frequenter enim quod arma explere nequeunt, oblectamenta suauitatis imponunt. Sit ergo pro re publica et cum ludere uidemur... Agnoscant per te exterae gentes tales nos habere nobiles, quales leguntur auctores. Quotiens non sunt credituri quae uide-

48 Cf. Gillett 2002a.

49 AMORY 1993; ID. 1994.

${ }^{50}$ L'attitude d'une partie de la noblesse d'origine gallo-romaine est révélatrice de la reconstruction des identités au $\mathrm{V}^{\mathrm{e}}$ puis au $\mathrm{VI}^{\mathrm{e}}$ siècle. En effet, le récit par Grégoire de Tours de la bataille de Vouillé montre que des Gallo-romains des plus grandes familles ont combattu au côté des Wisigoths (Greg. Tur. II, 37) en dépit de l'arianisme des rois wisigoths.

51 Léon dans l'entourage d'Euric (Ennod. Vita Epiph. 85-89; Sid. Apoll. Epist. IV, 22), Syagrius ou Aredius dans celui de Gondebaud (Sid. Apoll. Epist. V, 5; Greg. Tur. H.F., II, 32).

52 Sid. Apoll. Carm. VII, 495-498; Greg. Tur. H.F. II, 34. 
rint? Quotiens hanc ueritatem lusoria somnia putabunt? Et quando fuerint ab stupore conuersi, non audebunt se aequales nobis dicere...

Je [Théodoric] ne dois pas dédaigner les faveurs hardies demandées par les rois voisins pour plaire à leur vanité, puisque la plupart du temps, une petite chose peut souvent obtenir plus que de grandes richesses. En effet, souvent ce que les armes n'imposent pas, l'amusement et les douceurs l'imposent. Ainsi je sers la res publica même si je semble jouer... Faites reconnaître par les gentes externes de ce genre que nos nobles envoyés en légation sont de telles autorités. Combien de fois ne croiront-ils pas ce qu'ils voient? (...) Et quand ils seront revenus de leur stupeur, ils n'oseront plus se dire égaux à nous... 53

L'emploi de la première personne du pluriel par Cassiodore est particulièrement intéressant: il affirme la supériorité du roi ostrogoth par rapport aux autres rois barbares en l'incluant dans la Romanitas. L'influence des conseillers romains, particulièrement de ceux issus de l'aristocratie, est indéniable dans cette approche qui fait de l'accès à la culture latine, une forme de supériorité politique pour les rois barbares. Et finalement, le pragmatisme des aristocrates romains leur a permis de réussir, en imposant la culture latine comme moyen d'affirmation politique, à rester indispensable sur le plan politique. ${ }^{54}$

\section{Conclusion}

C'est dans cette assimilation par les élites du groupe dominant politiquement, c'est-àdire les barbares, des valeurs culturelles du groupe dominé, c'est-à-dire l'aristocratie romaine, que réside le paradoxe anthropologique d'une situation en terme d'acculturation tout à fait particulière. Cette situation témoigne de la réussite de l'aristocratie romaine qui en se redéfinissant avant tout culturellement a élargi les critères d'inclusion dévalorisant en particulier le critère de l'origine ethnique avec non pas la fin d'une civilisation mais la redéfinition des élites des nouveaux royaumes barbares. ${ }^{55}$ Cette redéfinition en grande partie culturelle des élites remet dès lors en cause la pertinence d'une approche ethnique pour appréhender la construction de ces sociétés au $\mathrm{V}^{\mathrm{e}}$ siècle car cette frontière culturelle avec laquelle les élites romaines jouent n'envisage jamais comme critère primordial l'appartenance ethnique. Et c'est dès lors toutes les approches mettant en avant la primauté des critères ethniques dans la constitution des élites politiques des proto-états barbares qui se trouvent remises en cause. Certes, l'identité des individus en général, et plus particulièrement dans les aristocraties romaines ou barbares, comportait un élément ethnique que les individus

53 Cassiod. Var. I, 45.

54 Sur cette question, BECKER 2013, 190-213.

55 C'est en cela qu'il faut faire particulièrement attention à toute instrumentalisation de cette période de l'histoire pour justifier des visions contemporaines du monde en terme de choc des civilisations destructeurs pour une civilisation pensée comme, comme c'est le cas dans les ouvrages de S. Huntington qui n'hésite pas à interpréter l'histoire européenne de façon tout à fait fantaisiste, en particulier dans HunTINGTON 2004. Pour une critique très argumentée des théories de Huntington, CREPON 2002. 
eux-mêmes pouvaient revendiquer, mais celui-ci ne laissait pas nécessairement présager de leur allégeance politique. Et rien dans les sources littéraires contemporaines du $\mathrm{V}^{\mathrm{e}}$ siècle et du début du $\mathrm{VI}^{\mathrm{e}}$ siècle ne permet même d'affirmer que ce critère ait eu une quelconque importance lorsqu'il s'agissait pour les rois barbares de choisir leurs hommes de confiance. En revanche, l'accès à la culture latine a permis à ces rois, dans un second temps de se réapproprier les discours romains sur l'ethnicité dans les récits des origines à destination des élites des différents royaumes et visant, alors, à renforcer l'allégeance politique de ces élites au pouvoir en place au moment de la rédaction des différents récits à partir des $\mathrm{VI}^{\mathrm{e}}$ et $\mathrm{VII}^{\mathrm{e}}$ siècles. ${ }^{56}$

\section{BIBLIOGRAPHIE}

AMORY, P.

(1993): "The meaning and purpose of ethnic terminology in the Burgundian laws", EME 2, 1-28. (1994): "Names, ethnic identity and community in fifth- sixth-century Burgundy", Viator 25, 1-30.

(1997): People and Identity in Ostrogothic Italy, 489-554 (=Cambridge Studies in Medieval Life and Thought, Fourth Series, 33), Cambridge.

Armstrong, J. A. (1982): Nations before Nationalism, Chapell Hill.

BARTh, F. (1969): Ethnic Groups and Boundaries: The social organization of culture difference, Boston.

BAstenier, A. (2004): Qu'est-ce qu'une société ethnique? Ethnicité et racisme dans les sociétés européennes d'immigration, Paris.

Bauduin, P. (2008): “Introduction”, [in] Gazeau - Bauduin - Modéran (éd.), 2008, 7-19.

BECKER, A. (2013): Les relations diplomatiques romano-barbares en Occident au Ve siècle. Acteurs, fonctions, modalités, Paris.

BoudON, R.

(1979): La logique du social. Introduction à l'analyse sociologique, Paris.

(2002): “Théorie du choix rationnel ou individualisme méthodologique?", Sociologie et sociétés 34/1, 9-10 (http://dx.doi.org/10.7202/009743ar).

Bradley, D. R. (1966): “The Composition of the Getica”, Eranos 64, 67-69.

Brather, S. (2002): "Ethnic Identities as Constructions of Archeology: The Case of the Alamanni”, [in] Gillett (ed.), 2002, 149-175 (http://dx.doi.org/10.1484/M.SEM-EB.3.4487).

Brogiolo, G. P. - Chavarría Arnau, A. (2005): Aristocrazie e campagne nell'Occidente da Costantino a Carlo Magno (=Metodi e temi dell'archeologia medievale 1), Borgo San Lorenzo.

Carruthers, M. (1990): The Book of Memory. A Study of Memory in Medieval Culture (=Cambridge Studies in Medieval Literature 10), Cambridge.

Coleman, J. S. (1994): Foundations of social Theory, Cambridge-London.

56 Cf., par exemple, Coumert 2007, 143-264 qui étudie et compare deux récits des origines des Lombards l'Edit de Rothari et l'origo gentis Langobardorum. 
Coumert, M.

(2007): Origines des peuples, Les récits du Haut-Moyen Âge occidental (550-850), (=Collection des Études Augustiniennes, Série Moyen Âge et Temps Modernes 42), Paris.

(2008): “L'identité ethnique dans les récits d'origine: l'exemple des Goths", [in] Gazeau Bauduin - Modéran (éd.), 2008, 49-73.

Crepon, M. (2002): L'Imposture du choc des civilisations, Paris.

CurTa, F.

(2002): "From Kossina to Bromley: Ethnogenesis in Slavic Archaeology", [in] Gillett (ed.), 2002, 201-218 (http://dx.doi.org/10.1484/M.SEM-EB.3.4489).

(2013): "The Elephant in the Room. A Reply to Sebastian Brather", Ephemeris Napocensis XXIII, 163-174.

De Vos, G. A. - Romanucci-Ross, L. (eds.), (1975): Ethnic Identity: Cultural Continuities and Change, Mayfield.

Dragadze, T. (1980): "Soviet Ethnos Theory", Royal Anthropological Institute of Great Britain and Ireland 38, 3-4.

Gazeau, V. - Bauduin, P. - Modéran, Y. (ÉD.), (2008): Identité et Ethnicité, Concepts, débats historiographiques, exemples (III - XII ${ }^{e}$ siècle), (=Table ronde du Centre de Recherches Archéologiques et Historiques Médiévales 3), Caen.

Geary, P. J.

(1983): "Ethnic Identity as a Situational Construct in the Early Middle Ages", Mitteilungen der anthropologischen Gesellschaft in Wien 113, 15-26.

(2003): The Myth of the Nations, The Medieval Origins of Europe, Princeton.

Geertz, C. (1963): Old Societies, New States: the Quest for Modernity in Asia and Africa, New York.

Gillett, A.

(2002): "Ethnicity, History and Methodology", [in] Gillett (ed.), 2002, 1-18 (http://dx.doi. org/10.1484/M.SEM-EB.3.4481).

(2002a): "Was Ethnicity Politicized in the Earliest Medieval Kingdoms?", [in] Gillett (ed.), 2002, 85-122 (http://dx.doi.org/10.1484/M.SEM-EB.3.4485).

Gillett, A. (ED.), (2002): On Barbarian Identity, Critical Approaches to Ethnicity in the Early Middle Ages (=Studies in the Early Middle Ages 4), Turnhout.

Glazer, N. - Moynihan, D. P. (EDs.), (1975): Ethnicity: Theory and Experience, Cambridge (Mass.).

GOFFART, W.

(1988): The Narrators of Barbarians History (550 - 800), Jordanes, Gregory of Tours, Bede and Paul the Deacon, Princeton.

(2002): "Does the distant past impinge on the Invasion Age Germans?", [in] Gillett (ed.), 2002, 21-37 (http://dx.doi.org/10.1484/M.SEM-EB.3.4482).

(2006): Barbarian Tides, The Migration Age and the Later Roman Empire, Philadelphia.

Greatrex, G. (2000): “Introduction”, [in] Mitchell - Greatrex (eds.), 2000, xi-xvii.

Halsall, G.

(2007): Barbarian Migrations and the Roman West, 376-568, Cambridge.

(2011): "Ethnicity and early medieval cemeteries", Arqueología y Territorio Medieval 18, 15-27. 
HEATHER, P.

(1996): The Goths, Oxford.

(2003): "Gens and regnum among the Ostrogoths", [in] H.-W. Goetz - J. Jarnut - W. Pohl (eds.), Regna and Gentes. The Relationship between Late Antique and Early Medieval Peoples and Kingdoms in the Transformation of the Roman World (=Transformation of the Roman World 13), Leiden, 85-133.

Hornblower, S. - Spawforth, A. (Eds.), (2012): Oxford Classical Dictionnary, Oxford.

Hummer, J. (1998): "The Fluidity of Barbarian Identity: the Ethnogenesis of Alemanni and Suebi, AD 200-500”, EME 7, 1-27 (http://dx.doi.org/10.1111/1468-0254.00016).

Huntington, S. (2004): Who are we? The Challenges to America's National Identity, New York. IsAJIw, W. W. (1974): “Definitions of Ethnicity”, Ethnicity 1, 111-124.

Juteau, D. (1999): L'ethnicité et ses frontières, Montréal.

Kallen, H. M. (1924): Culture and Democracy in the United States, New York.

KazAnski, M. - PÉRIN, P. (2008): “Identité ethnique en Gaule à l'époque des Grandes Migrations et des Royaumes barbares: étude de cas archéologiques", Antiquités Nationales 39, 181-216.

Kосн, U. (1997): "Ethnische Vielfalt im Südwesten: Beobachtungen in merowingerzeitlichen Gräberfeldern an Neckar und Donau”, [in] K. Fuchs (ed.), Die Alamannen, Stuttgart, 219-232.

Leach, E. (1954): Political Systems of Highland Burma: a Study of Kachin Social Structure, London.

Martindale, J. R. (1980-1992): The Prosopography in the Later Roman Empire, vol. II (395527), vol. III (527-641), Cambridge.

McKitterick, R. (2004): History and Memory in the Carolingian World, Cambridge.

Mitchell, S. - Greatrex, G. (eds.), (2000): Ethnicity and Culture in Late Antiquity, London.

Moerman, M. (1968): "Being Lue: Uses and Abuses of Ethnic Identification", [in] J. Helm (ed.), Essays on the Problem of Tribe, Washington, 153-169.

Momigliano, A. (1955): “Cassiodorus and Italian Culture of His Time”, PBA 41, 207-245.

Naroll, R. (1964): “On ethnic unit classification”, Current Anthropology 5, 283-291 (http:// dx.doi.org/10.1086/200501).

Pallas-Brown, R. (2000): "East Roman perceptions of the Avars in the mid-and late-sixth century", [in] Mitchell - Greatrex (eds.), 2000, 309-330.

Patterson, O. (1975): “Context and Choice in Ethnic Allegiance: A Theoretical Framework and Caribbean Case Study", [in] Glazer - Moynihan (eds.), 1975, 305-349.

PoHL, W.

(1992): “Konfliktverlauf und Konfliktbewältigung: Römer und Barbaren im frühen Mittelalter", FMS 26, 165-207.

(2002): "Ethnicity, Theory and Tradition: A Response", [in] Gillett (ed.), 2002, 221-239 (http://dx.doi.org/10.1484/M.SEM-EB.3.4490).

(2005): “Aux origines d'une Europe ethnique. Transformations d'identités entre Antiquité et Moyen Âge", Annales. Histoire Sciences Sociales 60/1, 183-208.

(2006): "Telling the Difference: Signs of Ethnic Identity", [in] F. X. Noble (ed.), From Roman provinces to Medieval kingdoms, New York, 120-166.

(2008): "Nouvelles identités ehtniques entre Antiquité tardive et haut Moyen Âge", [in] Gazeau - Bauduin - Modéran (éd.), 2008, Caen, 23-33. 
PoHL, W. (ED.), (1997): Kingdoms of the Empire, The Integration of Barbarians in Late Antiquity (=Transformation of the Roman World 1), Leiden.

Pohl, W. - Reimitz, H. (EDs.), (1998): Strategies of Distinction, The Construction of Ethnic Communities, 300-800 (=Transformation of the Roman World 2), Leiden.

Poutignat, Ph. - Streiff-Fenart, J. (1995): Théories de l'ethnicité, Paris.

REYDELlEt, M. (1981): La royauté dans la littérature latine de Sidoine Apollinaire à Isidore de Séville (=Bibliothèque des Écoles françaises d'Athènes et de Rome 243), Rome.

Shanin, T. (1989): "Ethnicity in the Soviet Union: Analytical Perceptions and Political Strategies", Comparative Studies in Society and History 31, 409-423 (http://dx.doi.org/10.1017/ S0010417500015978).

Shils, E. (1957): "Primordial, Personal, Sacred and Civil Ties. Some Particular Observations on the Relationships of Sociological Research and Theory", The British Journal of Socio$\log y$ 8/2, 130-147.

SieGMUND, F.

(1996): "Kleidung und Bewaffnung der Männer im östlichen Frankenreich", [in] Die Franken: Wegbereiter Europas. Vor 1500 jahren: König Chlodwig und seine Erben, Mainz, vol. II, 691-706.

(1999): “Als Franke sterben: Ethnizität und Siedlungsraum der südlichen Nachbarn der Sachsen nach archäologischen Quellen”, Studien zur Sachsenforschung 12, 209-222.

Sмiтh, A. D.

(1981): The ethnic revival in the modern world, Cambridge.

(1984): "Review article: Ethnic Persistence and National Transformation", The British Journal of Sociology 35/3, 452-461.

(1986): The Ethnic Origins of Nations, Oxford.

(1993): “A Europe of Nations. Or the Nation of Europe?”, Journal of Peace Research 30/2, 129-135 (http://dx.doi.org/10.1177/0022343393030002001).

(1994): “The Politics of Culture: Ethnicity and Nationalism”, [in] T. Ingold (ed.), Companion Encyclopedia of Anthropology: Humanity, Citizen, and Social Life, London-New York, 706-733.

Sмiтh, M. G. (1982): "Ethnicity and Ethnic Groups in America: the View from Harvard", Ethnic and Racial Studies 5/1, 1-22 (http://dx.doi.org/10.1080/01419870.1982.9993357).

Tishrov, V. A. (1994): "Inventions and Manifestations of Ethno-nationalism in Soviet Academic and Public Discourse", [in] R. Borofsky - B. Dobson - M. Jarrett (eds.), Assessing Cultural Anthropology, New York, 443-452.

VAn den Berghe, P. (1970): Race and ethnicity. Essays in Comparative Sociology, New York.

Wenskus, R. (1961): Stammesbildung und Verfassung: Das Werden der frühmittelalterlichen Gentes, Köln.

WOLFram, H.

(1970): “The Shaping of the Early Medieval Kingdom”, Viator 1, 1-20 (http://dx.doi. org/10.1484/J.VIATOR.2.301705).

(1990): Histoire des Goths, Paris.

(1990a): "Le genre de l'origo gentis", RBPh 68/4, 789-801 (http://dx.doi.org/10.3406/ rbph.1990.3742). 
Wolfram, H. - Pohl, W. (HRSG.), (1990): Typen der Ethnogenese unter besonderer Berücksichtigung der Bayern (=Denkschriften der philosophisch-historischen Klasse 201; Veröffentlichungen der Kommission für Frühmittelalterforschung 12), Wien.

ZeIss, H. (1931): "Die geschichtliche Bedeutung der frühmittelalterlichen Archäologie", HJ 51, 297-306. 